\title{
Trop2 regulates the proliferation and differentiation of murine compact-bone derived MSCs
}

\author{
JIANYE YANG ${ }^{1,2}$, ZHAOHUI ZHU ${ }^{3}$, HONGFEI WANG ${ }^{2}$, FEIFEI LI ${ }^{2}$, XINLING DU ${ }^{2}$ and RUNLIN Z. MA ${ }^{3}$ \\ ${ }^{1}$ Department of Cardio-Thoracic Surgery, Tongji Hospital, Tongji Medical College, Huazhong University of \\ Science and Technology, Wuhan, Hubei 430030; ${ }^{2}$ Department of Cardiovascular Surgery, Union Hospital, \\ Tongji Medical College, Huazhong University of Science and Technology, Wuhan, Hubei 430022; \\ ${ }^{3}$ State Key Laboratory of Molecular and Developmental Biology, Institute of Genetics and \\ Developmental Biology, Chinese Academy of Sciences, Beijing 100101, P.R. China
}

Received April 8, 2013; Accepted June 3, 2013

DOI: $10.3892 /$ ijo.2013.1987

\begin{abstract}
Mesenchymal stem cells (MSCs) show promising potential in cell-based therapies for wound repair and tissue engineering. However, their applications are hindered by our limited understanding of the detailed regulation of the cell fates in vitro and in vivo. To investigate the relationship between MSCs and Trop2, a protein overexpressed in cancer cells, we constructed a Trop 2 gene knockout mouse by homologous recombination. Herein, we report that Trop2 is expressed exclusively on the MSC membrane and is involved in the regulation of proliferation and differentiation of MSCs. The proliferation of MSCs in Trop2 KO mice was significantly inhibited with prolonged cellular doubling time and reduced cell numbers entering the $\mathrm{S}$ phase. Trop2 deficiency impaired the differentiation of MSCs by significant reduction of adipogenesis and osteogenesis. AKT activation was impaired and cyclin D1 expression was downregulated in the Trop2-deficient MSCs, resulting in significant delay in cell cycle progression and inhibition of MSC self-renewal. Collectively, our findings help to establish a close functional link between Trop2 and MSCs, and to provide a tool for future investigation of Trop2 in other types of stem cells.
\end{abstract}

\section{Introduction}

Mesenchymal stem cells (MSCs) are a subgroup of stem cells with the capacity of osteogenesis, chondrogenesis, adipogenesis,

Correspondence to: Professor Xinling Du, Department of Cardiovascular Surgery, Union Hospital, Tongji Medical College, Huazhong University of Science and Technology, 1277 Jiefang Road, Wuhan, Hubei 430022, P.R. China

E-mail: xinlingdu616@hotmail.com

Professor Runlin Z. Ma, Center for Developmental Biology, Institute of Genetics and Developmental Biology, Chinese Academy of Sciences, Beijing 100101, P.R. China

E-mail: runlin.ma@hotmail.com

Key words: mesenchymal stem cells, Trop2, proliferation, differentiation, AKT/cyclin D1 pathway and myogenesis in adult organs and tissues (1-5). Although MSCs show promising potential in the stem-cell based therapies for wound repairing and tissue engineering $(6,7)$, the application is hindered due to our limited understanding on the detailed regulation of MSCs. Currently a combination of cell-surface markers has to be employed for routine identification of MSCs from other cell types (8).

Previous studies indicate that Trop2, a 36-kDa glycoprotein overexpressed in variety of epithelial cancers with unknown functions (9-14), could potentially serve as a stem-cell marker. Expressions of Trop2 were observed in progenitor/stem-celllike prostate basal and hepatic oval cells, and only those with a higher level of the expression were associated with a higher capacity of proliferation and differentiation $(15,16)$. These findings imply that Trop2 may be associated with certain functions of the stem-like cells, including self renewal of the cells.

To investigate the potential association between Trop2 and MSCs, we constructed a Trop2 knockout mouse by homologous recombination. We report that Trop2 is exclusively expressed on the MSCs membrane and is involved in regulation of proliferation and differentiation of MSCs. Trop2 deficiency impaired the differentiation of MSCs by significant reduction of adipogenesis and osteogenesis. AKT activation was impaired and cyclin D1 expression was downregulated in the Trop2-deficient MSCs, resulting in significant delay in cell cycle progression and inhibition of MSC self-renewal. These studies presented first-line evidence of close association between Trop2 and MSC self-renewal, providing a novel platform for further investigation of Trop2 on other types of stem cells.

\section{Materials and methods}

Trop2 gene knockout mice. Trop2 $\mathrm{KO}$ mice were generated by targeted gene interruption via homologous recombination (Fig. 1) at the Institute of Genetics and Developmental Biology, Chinese Academy of Sciences, following the modified protocol of Hall et al (17). Briefly, the full-length genomic sequence of Trop 2 was obtained by screening a mouse 129/ $\mathrm{Sv}$ bacterial artificial chromosome (BAC) library constructed 
Table I. Specific primers for RT-PCR amplification.

\begin{tabular}{llll}
\hline Marker & \multicolumn{1}{c}{ Forward $\left(5^{\prime} \rightarrow 3^{\prime}\right)$} & Reverse $\left(5^{\prime} \rightarrow 3^{\prime}\right)$ & Length $(\mathrm{bp})$ \\
\hline PPAR- $\gamma 2$ & ccgtgatggaagaccactcg & tcgcactttggtattcttggag & 165 \\
Osteocalcin & ctgctcactctgctgacctg & tcactaccttattgcctcctg & 118 \\
Osteopontin & tcaccattcggatgagtctg & acttgtggctctgatgttcc & 222 \\
Adipsin & tgggagcggctgtatgtg & agtcgtcatccgtcactccat & 189 \\
GAPDH & aacttggcattgtggaagg & ggatgcagggatgatgttct & 132 \\
\hline
\end{tabular}

Primer sequences, length (in base pairs) of amplified fragments is indicated. Respective length of each amplified fragment is given. The primers were designed with Primer Premier Software.

in the laboratory of Dr R.Z. Ma. ES cells of 129/Sv origin were a gift from Professor R. Xu at the Fudan University. Trop $2^{+/-}$heterozygous mice were used for breeding homozygous $\mathrm{KO}$ mice. All the animals were maintained in certified SPF facilities and the experiments were approved by the ethics committees for animal use and care at the Institute of Genetics and Developmental Biology, Chinese Academy of Sciences, and Tongji Medical College of Huazhong University of Science and Technology. The Trop2 KO mice (Trop2 $2^{-/}$) show no obvious phenotypes differences with the WT under normal conditions.

MSCs isolation and culture. Procedures of Zhu et al (18) were followed in isolation of the MSCs from the murine compact bone. Briefly, 2-3-week old WT and KO mice were sacrificed by cervical dislocation and all tissues on femur, humerus and tibia were removed. The epiphyseal end was cut and 20-gauge syringe was used to flush the bone marrow cavity with $\alpha$-MEM (Hyclone Laboratories, Logan, UT, USA). The bone fragments (cut in $1 \mathrm{~mm}^{3}$ ) were digested in $1 \mathrm{mg} / \mathrm{ml}$ type II Collagen (Gibco BRL, Grand Island, NY, USA) at $37^{\circ} \mathrm{C}$ for $90 \mathrm{~min}$ and then washed for 3-5 times in $\alpha$-MEM before seeding into a $25-\mathrm{cm}^{2}$ flask for cell culture in complete medium with $5 \% \mathrm{CO}_{2}$. The medium contains $\alpha$-MEM (Hyclone Laboratories), 10\% MSC-qualified FBS (Gibco BRL), 2 mM L-glutamine (Sigma-Aldrich, St. Louis, MO, USA), $100 \mathrm{U} / \mathrm{ml}$ penicillin (Sigma), and $100 \mu \mathrm{g} / \mathrm{ml}$ streptomycin (Sigma). Cells were digested for 2-3 min with $0.025 \%$ trypsin $/ 0.02 \%$ EDTA (Sigma) when reached $80 \%$ confluences for propagation. The passage was performed at a ratio of $1: 3$ or $1: 4$

Flow cytometry. Adherent cells from WT mice or Trop2 KO mice were digested and harvested for in vitro experiments. Cells were incubated with the anti-mouse monoclonal antibodies FITC-CD45, FITC-Sca-1, PE-CD34, PE-CD44, and PE-CD117 (all from eBioscience, San Diego, CA, USA) for $30 \mathrm{~min}$ at the room temperature. FITC or PE conjugated IgG was used in the control group. Cellular phenotypes were assessed by a FACSCalibur CellSorting System (BD Biosciences, Mountain View, CA, USA). For cell cycle analyses, the cells were fixed in pre-chilled $70 \%$ alcohol $\left(-20^{\circ} \mathrm{C}\right)$ for $24 \mathrm{~h}$ and then incubated with RNase A $(0.1 \mathrm{mg} / \mathrm{ml}$, Roche Applied Science, Basel, Switzerland) and propidium iodide $(20 \mu \mathrm{g} / \mathrm{ml}, \mathrm{Gibco})$ at $37^{\circ} \mathrm{C}$ for $25 \mathrm{~min}$. Detection channel FL1 or FL2 was used for the signal collection. CellQuest software (BD Biosciences) was utilized to analyze data.

Immunofluorescence. MSCs from WT and Trop2 KO mice were cultured on Polysine ${ }^{\mathrm{TM}}$ Microscopy Slides (Menzel-Gläser, Braunschweig, Germany) and fixed with $4 \%$ paraformaldehyde, then blocked with serum from non-immunized goats containing $0.3 \%$ Triton X-100. The cells were then incubated with diluted primary antibodies overnight at $4^{\circ} \mathrm{C}$. The antibodies used were Sca-1 (1:200; ab25195; Abcam, Cambridge, UK) and mTrop2 (1:40; AF1122; R\&D Systems, Minneapolis, MN, USA). The cells were washed and incubated with FITCor PE-conjugated secondary antibodies for $60 \mathrm{~min}$. DAPI (4',6-diamidino-2-phenylindole, Invitrogen) was used to stain the nucleus. The slides were visualized using a Nikon A1R laser scanning confocal microscopy (Digital Eclipse C1si; Nikon Corp., Tokyo, Japan).

Cell viability and CFSE assays. Colorimetric CCK-8 assays (Dojindo Laboratories, Kumamoto, Japan) were used to measure cell viability. Briefly, MSCs at a designated passage were seeded into 96 -well plates at 2000 cells/100 $\mu 1$ for culturing. At $0,24,48,72$, or $96 \mathrm{~h}$ of incubation time point, $10 \mu \mathrm{l}$ of CCK-8 solution was added followed by incubation in the dark for 2-3 h. The optical density (OD) was measured at $450 \mathrm{~nm}$ on a microplate spectrophotometer (Multscan; Thermo Scientific, Rockford, IL, USA).

For CFSE assay, cells $\left(2 \times 10^{6}\right)$ at passage 5 were harvested and mixed in $2.5 \mathrm{mM}$ CFSE (Dojindo Laboratories), followed by incubation at $37^{\circ} \mathrm{C}$ in the dark for $5-10 \mathrm{~min}$. The reaction was terminated with $10 \%$ FBS and seeded into $60-\mathrm{mm}$ flasks after washing. Flow cytometry was performed at 0,24 , 48, 72, or $96 \mathrm{~h}$ of incubation, and ModFit software (Mac3.1 SP2; Verity Software House, Toshan, ME, USA) was used for proliferation analyses.

Differentiation assays. MSCs at passage 5 from WT mice and Trop2 $\mathrm{KO}$ mice were harvested and seeded into a 24-well plate at a density of $5 \times 10^{3} / \mathrm{cm}^{2}$ and then cultured in condition medium for adipogenesis or osteogenesis induction $\sim 2-3$ weeks, respectively. For adipogenesis induction the medium contains $\alpha$-MEM, $10 \%$ FBS, $10^{-3} \mathrm{mM}$ dexamethasone, $0.5 \mathrm{mM}$ IBMX (Sigma), and $10 \mathrm{ng} / \mathrm{ml}$ insulin (Sigma); while for osteogenesis the medium contains $\alpha$-MEM, $10 \% \mathrm{FBS}, 10^{-4}$ $\mathrm{mM}$ dexamethasone (Sigma), $10 \mathrm{mM} \beta$-glycerol phosphate 


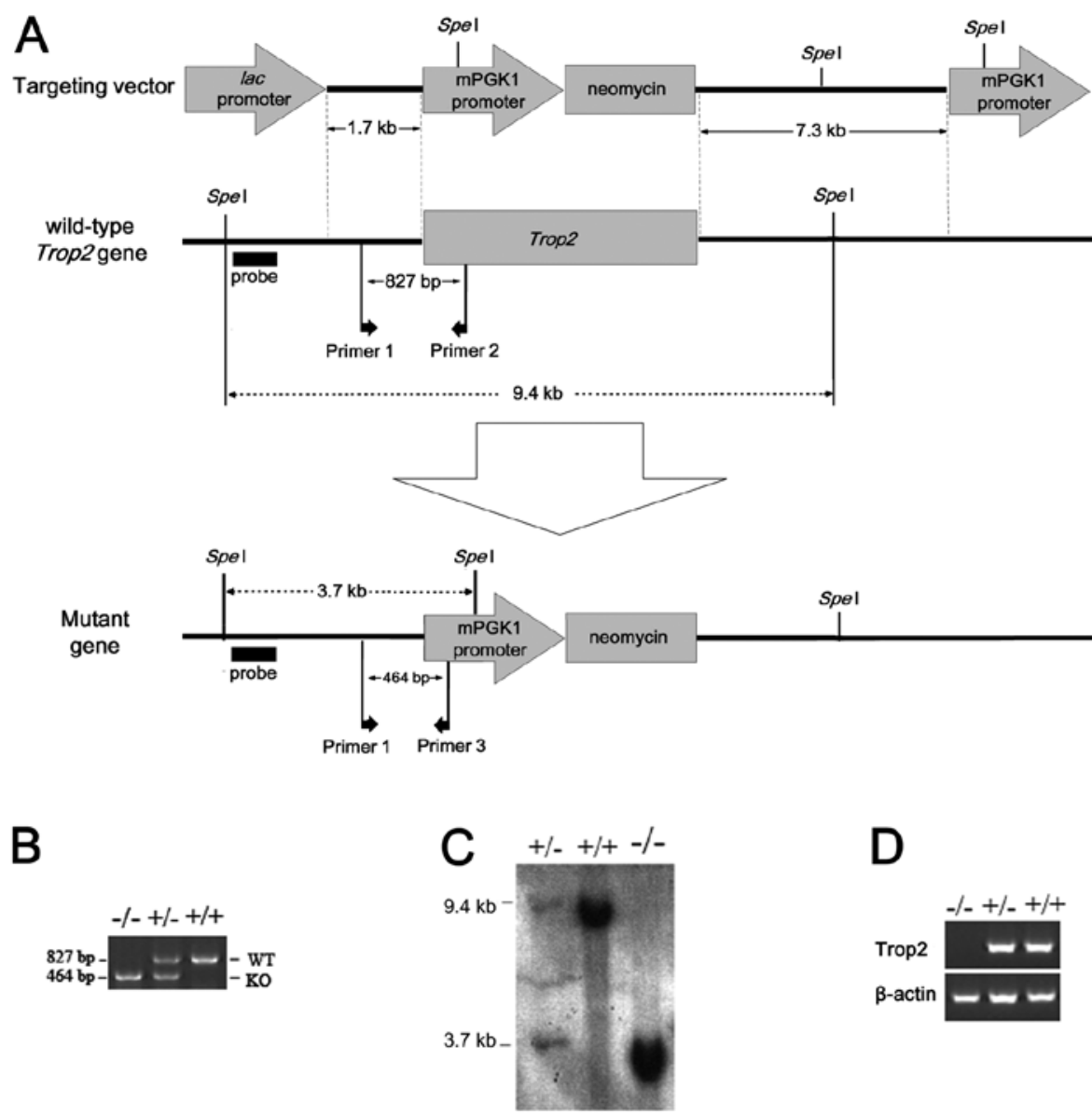

Figure 1. Construction of Trop 2 knockout mice by the targeted gene interruption. The whole ORF of Trop2 was replaced by a piece of DNA fragment coding for mPGK1 promoter-Neomycin via homologous recombination. (A) Experimental design strategy for the homologous recombination, with locations of target and replacement fragments, SpeI restriction sites, probes, primers, short arms and long arms marked as shown. (B) Genotype identification of Trop2 homozygous, heterozygous, and wild-type mice by SP-PCR. Genomic DNA isolated from the individual mouse tail was used as templates for routine mouse colony screening. WT: Wild-type, KO: Knockout. (C) Southern blot analysis of mice genomic DNA digested with SpeI restriction enzyme, showing the expected ${ }^{32} \mathrm{P}$-labeled Trop2 band in wild-type, heterozygous, and homozygous mouse. (D) PCR amplification of Trop2 cDNA reverse transcribed from mRNA of WT, heterozygous, or homozygous. No Trop 2 mRNA was detected in homozygous mice, indicating successful knockout of the target gene.

(Sigma), and $50 \mu \mathrm{M}$ ascorbic acid (Sigma). For adipogenesis induction the cells were fixed with $4 \%$ paraformaldehyde $\left(4^{\circ} \mathrm{C}\right)$ for $1 \mathrm{~h}$ and stained with oil red $\mathrm{O}$ for $30 \mathrm{~min}$; for osteogenesis induction the cells were fixed in pre-chilled $70 \%$ alcohol $\left(4^{\circ} \mathrm{C}\right)$ for $1 \mathrm{~h}$ and then stained with alizarin red at room temperature for $15 \mathrm{~min}$. After removing the staining solutions, and rinsing, representative images were captured.

Real-time RT-PCR. Following induction of osteogenesis or adipogenesis, MSCs from WT and Trop2 KO mice were harvested and total RNA was extracted using an RNeasy RNA isolation kit (Qiagen, Hilden, Germany). DNase-treated total RNA $(2 \mu \mathrm{g})$ underwent reverse transcription using oligo (dT) $)_{12-18}$ as a primer and Superscript II RNase reverse transcriptase. cDNA was used as a template for the amplification of target genes by PCR using La-Taq and GC buffer I (Takara Bio, Shiga, Japan). The primers for PPAR- $\gamma 2$, Osteocalcin, Osteopontin, Adipsin and GAPDH (glyceraldehyde-3-phosphate dehydrogenase) are shown in Table I and were designed with Primer Premier Software (Premier Biosoft International, Palo Alto, CA, USA). Statistical analysis was performed with a Student's two-tailed test. These experiments were performed in triplicate.
Western blotting. MSCs in the logarithmic phase at the designated passage were collected and lysed on ice in PhosphoSafe Extraction Reagent (Merck KGaA, Darmstadt, Germany). Proteins in the lysate were resolved on 8-10\% SDS-polyacrylamide gels, transferred to PVDF membranes, and subjected for western blot analyses using the primary antibodies for Trop2 (1:600; AF1122; R\&D Systems), Akt (1:500; MAB2055; R\&D Systems), Phospho-Akt (Ser473, 1:1000; \#9271; Cell Signaling Technology, Beverly, MA, USA), and cyclin D1 (1:500; sc-56302; Santa Cruz Biotechnology, Santa Cruz,CA,USA). The blots were incubated with HRP-conjugated secondary IgG antibodies (Bio-Rad Laboratories, Hercules, CA, USA) and the images were captured and analyzed using a UVP AutoChem Image System (UVP, Upland, CA, USA).

\section{Results}

Breeding of the Trop2 $\mathrm{KO}$ mice. We successfully generated the Trop2 knockout mice (Fig. 1B-D). Initial analysis showed no significant differences in growth, development and reproduction between WT and Trop2 homozygous (Trop $2^{-\digamma}$ ) mice under normal SPF condition. However, we noted that when both WT and KO mice were placed under heat, humidity, and 

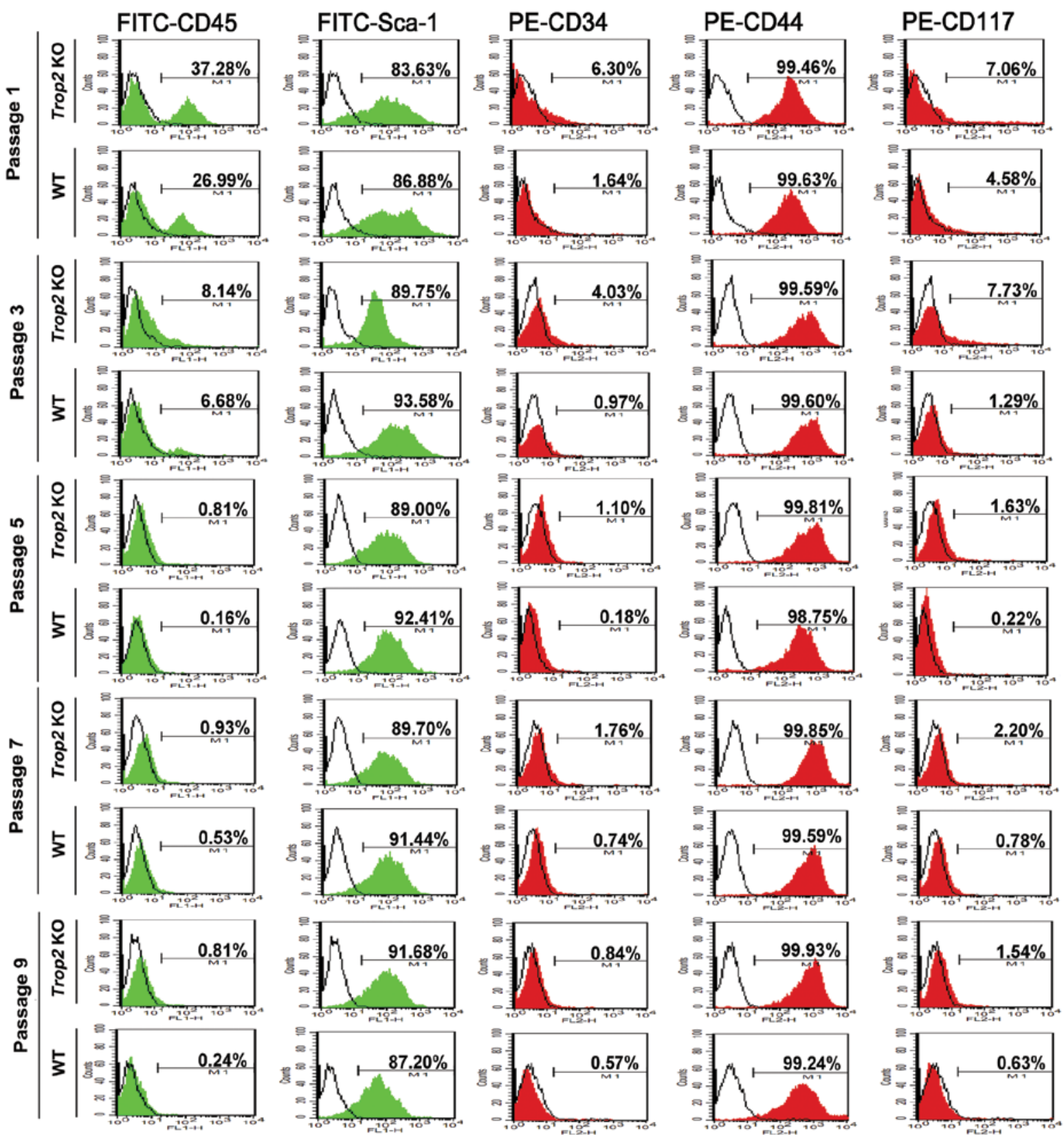

Figure 2. Phenotype analysis of compact-bone derived MSCs from WT and KO mice. Cell surface markers FITC-CD45, FITC-Sca-1, PE-CD34, PE-CD44, and PE-CD117 were selected to characterize the phenotypes of compact-bone derived MSCs from Trop2 KO and WT mice at the indicated passages by flow cytometry. Compared to WT, MSCs from KO mice showed significantly higher expression for CD45 (37.90 $\pm 3.45 \%$ vs. 27.50 $\pm 2.33 \%, \mathrm{n}=3 ; \mathrm{P}<0.05)$ and $\mathrm{CD} 34$ $(6.79 \pm 1.65 \%$ vs. $1.60 \pm 0.83 \%, \mathrm{n}=3 ; \mathrm{P}<0.05)$ at passage 1 , illustrating a significantly fewer compact-bone derived MSCs from Trop $2 \mathrm{KO}$ mice. The levels of CD45 and CD34 expression decreased along with the progression of cell passages, indicating an increasing purity of compact-bone derived MSCs over passages due to gradual elimination of hematopoietic cells. The red and green colored peak represents the specific PE-labeled antibodies (CD34, CD44 and CD117) and FITC-labeled antibodies (CD45 and Sca-1), respectively. The blank peak represents the corresponding isotope antibodies.

other stress conditions, the life expectancy of Trop2 KO mice was significantly reduced (data not shown).

Trop2 deficiency suppresses the total MSC number in the compact bone. Phenotype analysis of the compact-bone derived MSCs with 5 cell surface markers by flow cytometry showed the successful isolation of the compact bone-derived stem cells from both of the WT and Trop2 KO mice (Fig. 2). The MSC-positive rate for FITC-CD45 and PE-CD34 was significantly higher in WT $(\mathrm{CD} 45=37.28 \%, \mathrm{CD} 34=6.30 \%)$ than in Trop $2 \mathrm{KO}(\mathrm{CD} 45=26.99 \%, \mathrm{CD} 34=1.64 \%)$ for passage 1, demonstrating Trop2 deficiency significantly inhibited the total MSC number in the compact bone (Fig. 2). With progression of the primary cell cultures to passage 5 and higher, the differences between WT and KO MSCs become non-significant, due to the continuous differentiation of the MSCs under the culture conditions.

Trop2 is exclusively expressed on the MSC membrane. Immunofluorescence staining of the WT and KO derived MSCs using the specific anti-mouse Trop2 antibody illustrated that, intensive Trop2 fluorescence was localized exclusively on 


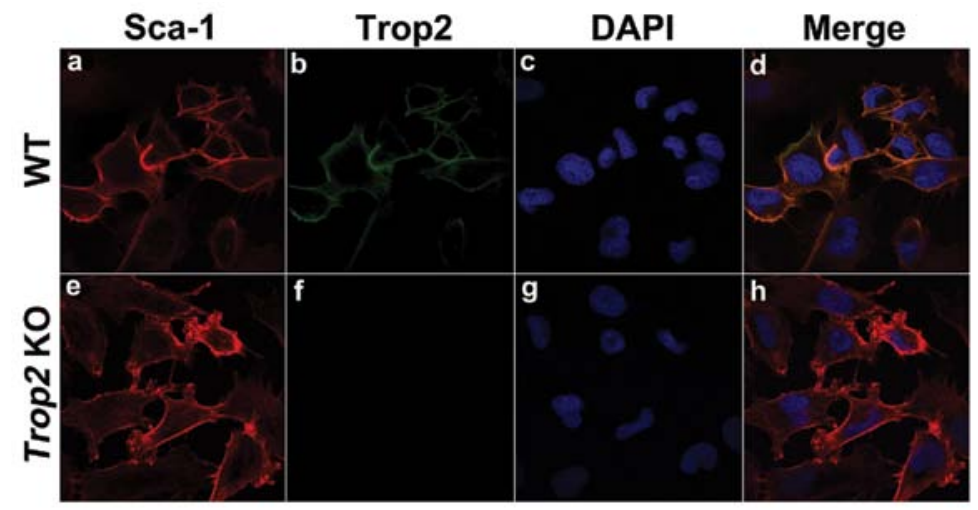

Figure 3. Localization of Trop 2 on the MSC membrane. Immunofluorescence assay with confocal microscopy (x400) was performed for the compact-bone derived MSCs from the WT (a-d) and the KO mice (e-h), using anti-Sca-1 antibodies (1:200; ab25195; Abcam, Cambridge) (red) and anti-Trop2 antibodies (1:40; AF1122; R\&D Systems, Minneapolis, MN, USA) (green). Expression of Trop2 was localized exclusively on the cell membrane of MSCs from WT mice (b). In contrast, no Trop2 signal was detected from MSCs of KO mice (f), indicating the complete elimination of the target protein in KO mice. Detection of Sca-1 on the MSC membrane served as a reference for the experimental system. The nucleus was stained with DAPI (blue). (d) Merged image of (a-c); (h) merged image of (e-g).
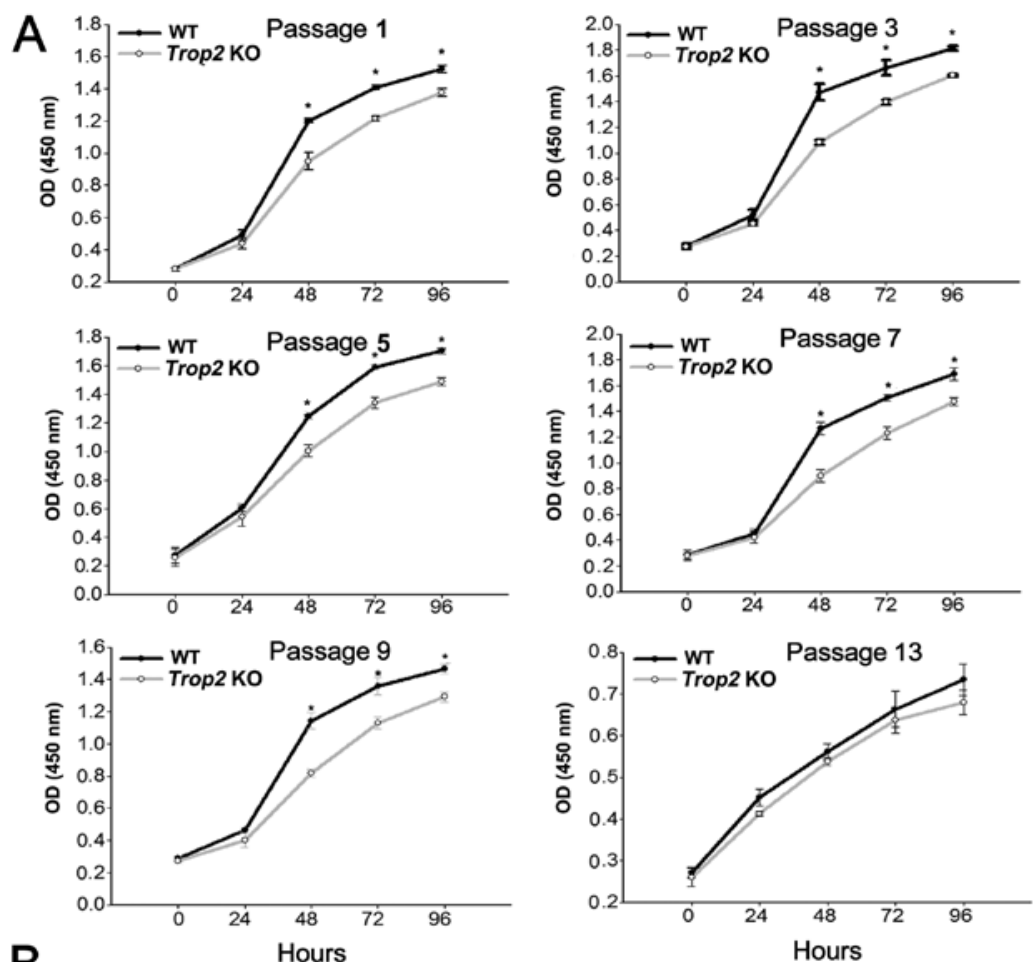

B

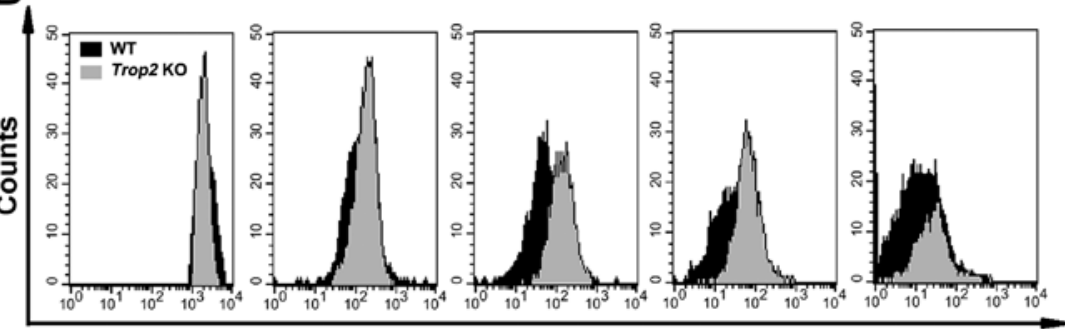

CFSE Fluorescence (FL1-H)

Figure 4. Absence of Trop2 impairs proliferation of compact-bone derived MSCs. (A) CCK-8 assay of MSCs after different passages of the cultures. For both KO and WT derived MSCs at the designated passages, equal amounts of the cells (2000 cells/100 $\mu$ l) were seeded in flat-bottomed 96-well plates at a final volume of $100 \mu 1$. The cell proliferation (total viable cell numbers) in each well was estimated by optical density (450 nm) using a Multiscan microplate spectrophotometer (Thermo Scientific, Rockford, IL, USA) at 0, 24, 48, 72, and $96 \mathrm{~h}$, after initial incubation for $3 \mathrm{~h}$. Except for the passage 13 (the last passage), MSCs from Trop $2 \mathrm{KO}$ mice showed a significantly decreased viable cell number than that of WT for the assays after $48 \mathrm{~h}$ of culture ( $\mathrm{n}=3$, $\left.{ }^{*} \mathrm{P}<0.01\right)$. (B) CFSE cellular doubling time analyses. MSCs premixed with CFSE from both of the KO and WT mice at the passage 5 were harvested and sorted by flow cytometry for CFSE decay at $0,24,48,72$, and $96 \mathrm{~h}$ of culturing. The detected fluorescence between KO and WT MSCs at each time point was compared and composed using ModFit software (Mac 3.1 SP2; Verity Software House, Toshan, ME, USA). Compared with WT (black peak area), Trop2 KO MSCs have a significantly longer cellular doubling time (grey peak area) after $48 \mathrm{~h}$ of culturing. 

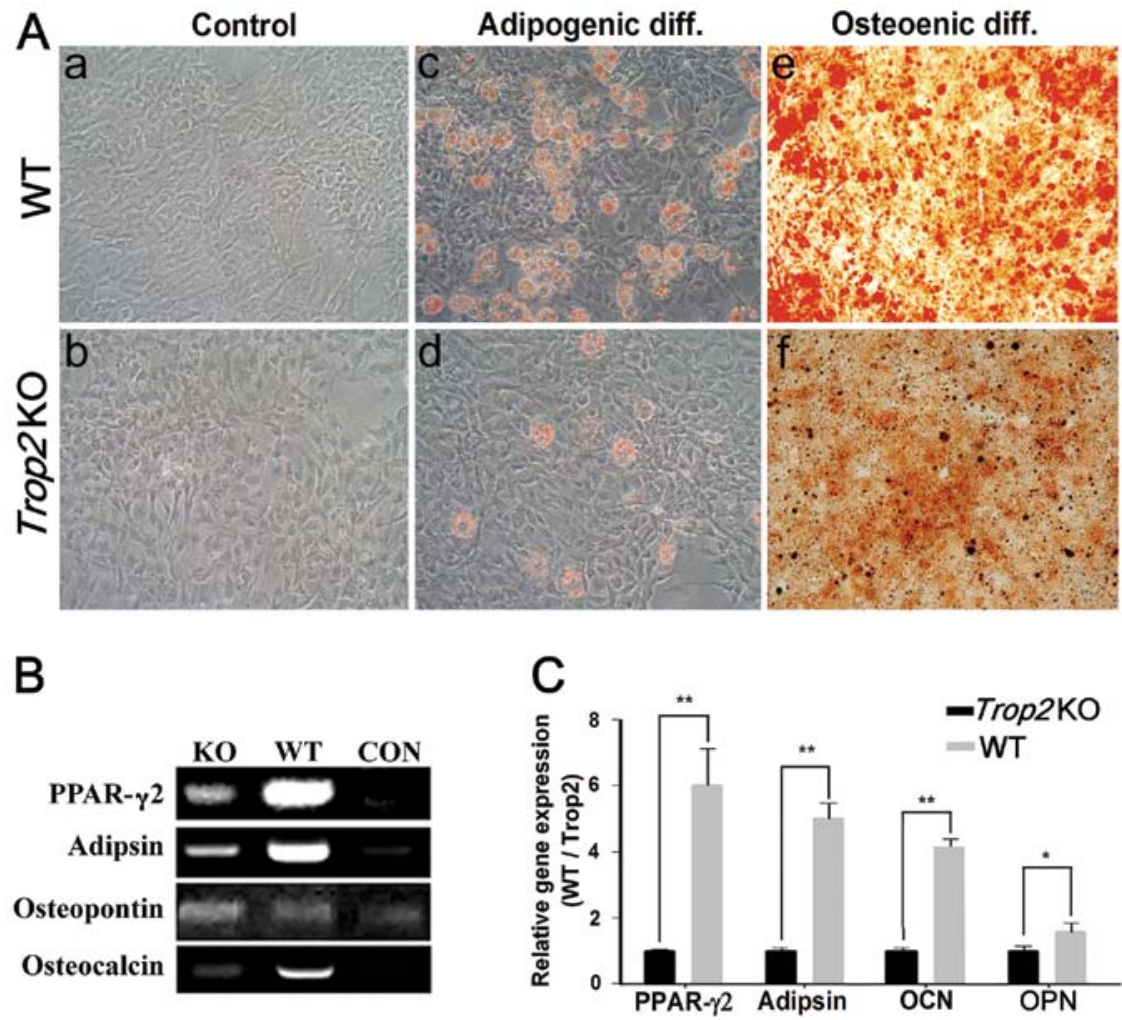

Figure 5. Absence of Trop2 impairs differentiation of compact-bone derived MSCs. (A) Adipogenesis and osteogenesis were impaired in Trop2 KO MSCs. Equal amounts of the cells from the KO and WT mice were cultured under the conditioned medium for 3-4 weeks, and the cell differentiation was estimated by Red Oil O and Alizarin Red staining for adipogenesis and osteogenesis, respectively. The untreated MSCs from the KO and WT mice were found negative for both adipogenesis and osteogenesis (a and b). In contrast, a significantly higher percentage of adipogenesis was observed in MSCs of WT (c) than that of Trop2 KO (d). Similarly, osteogenesis differentiation in MSCs of WT (e) was significantly higher than that of KO (f). (B and C) mRNA expression levels measured by RT-qPCR on WT and Trop2 KO MSCs of the marker genes were specifically involved in the process of adipogenesis (PPAR- $\gamma 2$ and Adipson) and osteogenesis (Osteocalcin and Osteopontin) were significantly decreased in the MSCs of Trop2 KO mice $\left(\mathrm{n}=3 ;{ }^{*} \mathrm{P}<0.05 ;{ }^{* * *} \mathrm{P}<0.01\right)$.

the cell membrane of MSCs from WT mice (Fig. 3), which was colocalized with Sca-1, a surface marker of MSCs (Fig. 3b and a). Trop2 fluorescence was absent on MSCs derived from the Trop2 KO mice, although Sca-1 fluorescence was present (Fig. $3 \mathrm{e}$ and f).

Absence of Trop 2 impairs the MSC proliferation. Measurement of the cell viability using CCK-8 assays showed that, the MSCs isolated from Trop2 KO mice exhibited a decreased viable cell number compared to WT, and the difference was maintained significant $(\mathrm{P}<0.05)$ throughout the assays except at passage 13 (Fig. 4A). Determination of MSC doubling time using the CFSE labeling showed that, Trop2 KO MSCs had a significantly prolonged cellular doubling time after $48 \mathrm{~h}$ of culturing as compared to that of the WT MSCs (Fig. 4B). These results indicated that Trop 2 deficiency leads to a reduction in MSC growth rate or the cell proliferation, via reduced cell viability and a prolonged cellular doubling time.

Cell differentiation is inhibited in the Trop2-deficient MSCs. Induction of cell differentiation showed that, compared to the MSCs from the WT mice, the process of adipogenesis and osteogenesis in the Trop2-deficient MSCs was significantly hindered, exhibited considerably less aggregation of lipid drops and mineral deposition for the MSCs at passage 5 (Fig. 5). In contrast, a significantly higher percentage of adipogenesis was observed in MSCs derived from the WT mice (Fig. 5A-c). Similarly, osteogenesis differentiation in MSCs of WT (Fig. 5A-e) was significantly higher than that of KO (Fig. 5A-f). Accordingly, mRNA transcription of the marker genes for the adipogenesis (PPAR- $\gamma 2$ and Adipson) and osteogenesis (Osteocalcin and Osteopontin) was dramatically decreased in the MSCs of Trop2 KO mice. ( $\mathrm{n}=3 ; \mathrm{P}<0.05$; $\mathrm{P}<0.01$ ) (Fig. 5B and C).

Trop2 deficiency impairs the AKT activation, Cyclin D1 expression, and cell cycle progression. MSCs from the WT and Trop $2 \mathrm{KO}$ mice were stained with propidium iodide at different time points, and their cell cycle distribution was measured by flow cytometry for the designated cell passages. Compared with the MSCs of the WT, the proportion of MSCs entering the $\mathrm{S}$ phase from the Trop2 KO mouse was significantly reduced at each passage $(\mathrm{P}<0.05$, Fig. $6 \mathrm{~A})$.

MSCs of the Trop2 KO mice have markedly lower expression for both of activated AKT and its downstream target Cyclin D1 at the same passages $(\mathrm{n}=3, \mathrm{P}<0.01$, Fig. $6 \mathrm{~B})$, indicating the AKT/Cyclin D1 pathway was impaired. In addition, activated AKT (p-AKT) expression in MSCs from both WT and Trop2 KO mice had a decreasing trend with the increase of passage, and the proportion of p-AKT in MSCs from WT mice was positively correlated with the reduction of Trop2 with the increase of passage. 


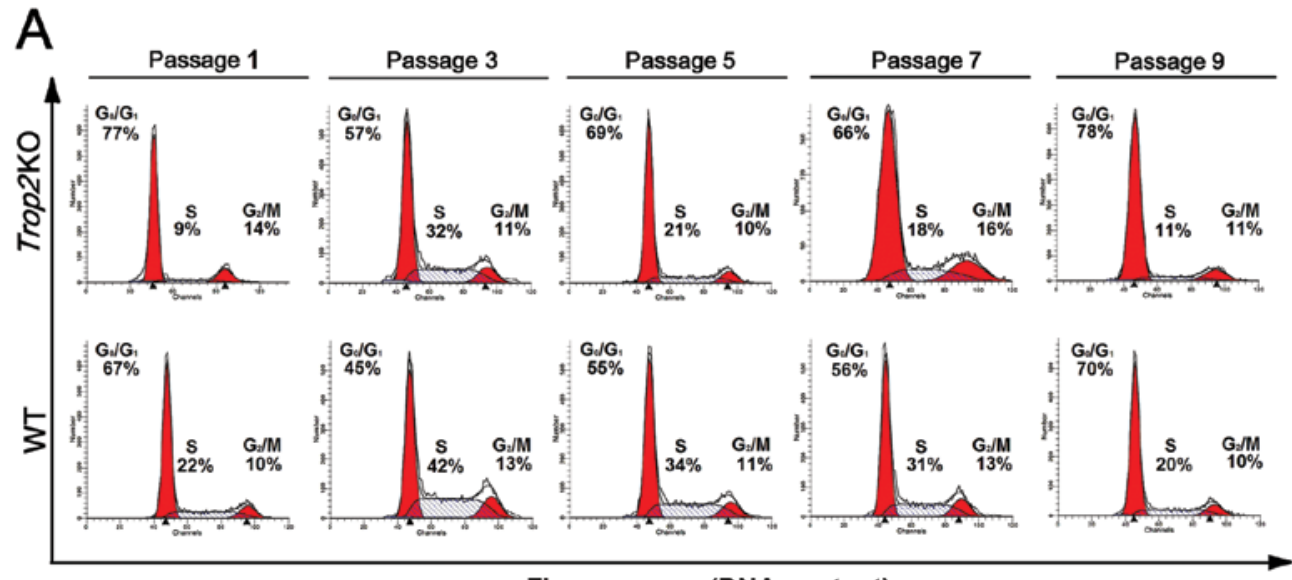

Fluorescence (DNA content)

B

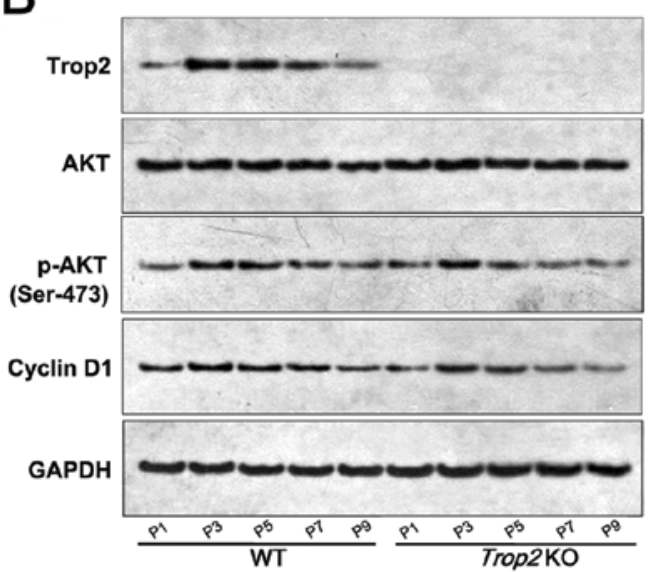

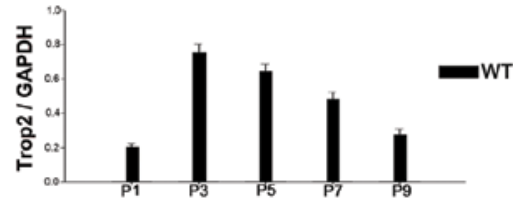
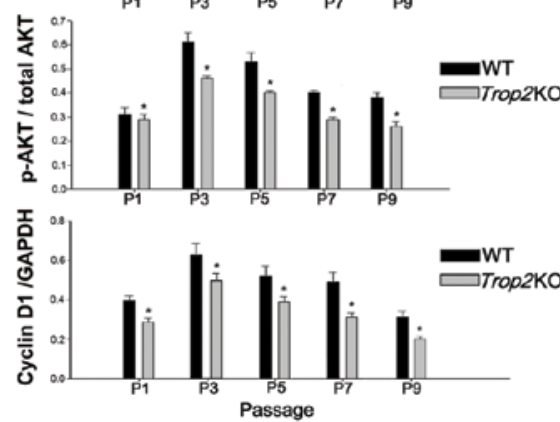

Figure 6. Trop2 deficiency impairs AKT activation, Cyclin D1 expression, and cell cycle progression. (A) Cell cycle analysis of the MSCs cultures at different passages. WT and Trop2 KO mouse compact bone-derived MSCs after different passages of cultures were collected, fixed, stained with propidium iodide and analyzed for cell cycle phase distribution. Compared with WT, the proportion of MSCs entering the S phase from Trop2 KO mice was significantly reduced compared to that of WT at the same passage $\left(n=3,{ }^{*} \mathrm{P}<0.01\right)$, indicating that the cell cycle progression was hindered in the MSCs from KO mice. (B) Western blot analysis of the cells at different passages. MSCs cultures at different passages were used to prepare cell lysates and the total cellular proteins were used for immunoblotting by antibodies against Trop2 (1:600; AF1122; R\&D Systems, Minneapolis, MN, USA), phosphorylated AKT (Ser473, 1:1000; \#9271; Cell Signaling Technology, Beverly, MA, USA), total AKT (1:500; MAB2055; R\&D Systems) and Cyclin D1 (1:500; sc-56302; Santa Cruz Biotechnology, Santa Cruz, CA, USA). No Trop2 expression was detected in MSCs from KO mice at any passage. Compared with WT, MSCs from Trop2 KO mice have markedly lower expression for both activated AKT and its downstream target cyclin D1 at same passages of cultures (n=3, $\mathrm{P}<0.01$ ), indicating that AKT/Cyclin D1 pathway of MSCs from KO mice was impaired.

These results imply that Trop2 deficiency impaired AKT phosphorylation and may have induced a cascade reaction, leading to downregulation of cyclin D1, and thus resulting in hindering cell cycle progression, which ultimately impaired proliferation and differentiation of the MSCs in vitro.

\section{Discussion}

In this study, we constructed the Trop2 $\mathrm{KO}$ mice and investigated the roles of Trop 2 on the proliferation and differentiation of murine compact bone-derived MSCs in vitro. We showed that Trop2 was exclusively localized on the MSC cell membrane; Trop2 deficiency impaired the proliferation, differentiation, cell cycle progression of the compact-bone derived MSCs, probably via partial inhibition of AKT/Cyclin D1 pathway. These results demonstrated the importance of Trop2 for the normal function of MSCs. Although the molecular details on how exactly the Trop2 interacts with the AKT/Cyclin D1 signal pathway remains unknown, our results may help to establish a novel platform for the future investigation.

Successful isolation and expansion of the primary MSCs from the compact-bone of both WT and KO mice enabled us to carry out the subsequent investigation. Previous studies showed that MSCs isolated from compact-bone were less contaminated with hematopoietic cells and had a better homogeneity compared to those isolated from bone marrow (18-20). Our results demonstrated that MSCs prior to the first 3 passages were inevitably contaminated by hematopoietic cells, but the cells after passage 4 reached much higher homogeneity with typical phenotypes of MSCs.

The result that Trop2 deficiency impaired the adipogenesis and osteogenesis of the MSCs have profound significance. Our data showed that the inhibition was due to the downregulation of the marker genes involved in both processes (Fig. 5). This fact indicates that one of the biological functions of Trop2 is to mediate an unknown signal pathway to maintain the proper 
function of the MSCs. Since it is critical for MSCs to maintain the differentiation potential for wound repairing and tissue regeneration $(21,22)$, any future clinical application of MSCs for wound repairing or tissue engineering would require the presence of Trop2 as a factor for the proper function of the stem cells. Further investigation of the mechanism of the downregulation would help to resolve the puzzle.

The specific ligand of Trop2 has not been identified, and thus the specific pathway in which Trop2 functions remains unclear (23). Trop2 is involved in signal transduction in that it possesses the $\mathrm{PIP}_{2}$ binding motif and tyrosine/serine phosphorylation sites (24-26). The presence of a $\mathrm{PIP}_{2}$ motif may promote the aggregation of PIP 2 on both sides of the cell membrane. This in turn could increase the probability of $\mathrm{PIP}_{2}$ hydrolysis, leading to an increased PIP3 level (23). PIP3 is involved in the activation of the PI3K/AKT signaling pathway $(27,28)$, which is a pivotal signaling pathway involved in the regulation of cell survival (29-31). We thus speculate that Trop2 may be helpful in the maintenance of AKT phosphorylation and is likely involved in AKT activation, which stabilizes the dephosphorylation of cyclin D1, resulting in promoting cell cycle progression and maintaining self-renewal potential.

The result that the proportion of active AKT in MSCs from Trop2 $\mathrm{KO}$ mice was significantly lower than that from WT mice suggested that Trop2 deficiency impaired the activation of AKT. However, we noted that the ratio of p-AKT/AKT and expression of cyclin D1 showed a tread of decline with the progression of the MSC culture passage, and the same was true for Trop2 expression except in passage 1, which was affected by hematopoietic cell contamination. This may be attributed to the fact of no addition of cytokines for both promoting proliferation and maintaining of undifferentiated state of MSCs during our subculture $(32,33)$. Without addition of the cytokines, MSCs became senescent and lost the potential of self-renewal, accompanied by gradually reduced expression of Trop2 during the passage. Thus, Trop2 may protect MSCs from aging during the passage, while the absence of Trop2 may promote MSCs senescence, which would subsequently impair the self-renewal potential of MSCs. However, Trop2 loss may not completely block AKT activation. On one hand, Trop2 deficiency cannot completely abolish AKT activation by some unknown cytokines in the MSC-qualified fetal bovine serum, or AKT may be activated in multiple pathways through complex signal networks (34). On the other hand, Trop2 may have pleiotropic functions as well as EpCAM, which regulates both adhesion (35), and intracellular signaling via cleavage products (36). Latest study revealed that Trop2 loss promoted the tumor aggressiveness and epithelial-mesenchymal transdifferentiation (37); while previous studies emphasized that Trop2 expression was positively related to the tumor aggressiveness and metastasis. Hence, further studies are required to elucidate the relationships between Trop2 and related signaling pathways in MSCs or other types of stem cells.

\section{Acknowledgements}

We thank Rene $\mathrm{Xu}$ and Yuan Zhuang for assistance in construction of the Trop2 KO mice. This study was supported by grants from National Natural Science Foundation of China (30571053; 30571840; 30771148), Specialized Research Fund for the Doctoral Program of Higher Education (20110142110009), and Chinese Academy of Sciences (KSCX1-YW-R-45; KSCX2-EW-R-05).

\section{References}

1. Caplan AI: Mesenchymal stem cells. J Orthop Res 9: 641-650, 1991.

2. Friedenstein AJ, Gorskaja JF and Kulagina NN: Fibroblast precursors in normal and irradiated mouse hematopoietic organs. Exp Hematol 4: 267-274, 1976.

3. Pittenger MF, Mackay AM, Beck SC, et al: Multilineage potential of adult human mesenchymal stem cells. Science 284: 143-147, 1999.

4. Jiang Y, Jahagirdar BN, Reinhardt RL, et al: Pluripotency of mesenchymal stem cells derived from adult marrow. Nature 418: 41-49, 2002.

5. Lee OK, Kuo TK, Chen WM, Lee KD, Hsieh SL and Chen TH: Isolation of multipotent mesenchymal stem cells from umbilical cord blood. Blood 103: 1669-1675, 2004.

6. Song H, Song BW, Cha MJ, Choi IG and Hwang KC: Modification of mesenchymal stem cells for cardiac regeneration. Expert Opin Biol Ther 10: 309-319, 2010.

7. Mizuno H: Adipose-derived stem cells for tissue repair and regeneration: ten years of research and a literature review. J Nippon Med Sch 76: 56-66, 2009.

8. Bianco P, Robey PG and Simmons PJ: Mesenchymal stem cells: revisiting history, concepts, and assays. Cell Stem Cell 2: 313-319, 2008.

9. Fong D, Moser P, Krammel C, et al: High expression of TROP2 correlates with poor prognosis in pancreatic cancer. Br J Cancer 99: 1290-1295, 2008.

10. Fong D, Spizzo G, Gostner JM, et al: TROP2: a novel prognostic marker in squamous cell carcinoma of the oral cavity. Mod Pathol 21: 186-191, 2008.

11. Muhlmann G, Spizzo G, Gostner J, et al: TROP2 expression as prognostic marker for gastric carcinoma. J Clin Pathol 62: $152-158,2009$.

12. Nakashima K, Shimada H, Ochiai T, et al: Serological identification of TROP2 by recombinant cDNA expression cloning using sera of patients with esophageal squamous cell carcinoma. Int J Cancer 112: 1029-1035, 2004

13. Ohmachi T, Tanaka F, Mimori K, Inoue H, Yanaga K and Mori M: Clinical significance of TROP2 expression in colorectal cancer. Clin Cancer Res 12: 3057-3063, 2006.

14. Wang J, Day R, Dong Y, Weintraub SJ and Michel L: Identification of Trop-2 as an oncogene and an attractive therapeutic target in colon cancers. Mol Cancer Ther 7: 280-285, 2008.

15. Goldstein AS, Lawson DA, Cheng D, Sun W, Garraway IP and Witte ON: Trop2 identifies a subpopulation of murine and human prostate basal cells with stem cell characteristics. Proc Natl Acad Sci USA 105: 20882-20887, 2008.

16. Okabe M, Tsukahara Y, Tanaka M, et al: Potential hepatic stem cells reside in $\mathrm{EpCAM}^{+}$cells of normal and injured mouse liver. Development 136: 1951-1960, 2009.

17. Hall B, Limaye A and Kulkarni AB: Overview: generation of gene knockout mice. Curr Protoc Cell Biol, Chapter 19: Unit 19.12 19.12.1-17, 2009. doi: 10.1002/0471143030.cb1912s44.

18. Zhu H, Guo ZK, Jiang XX, et al: A protocol for isolation and culture of mesenchymal stem cells from mouse compact bone. Nat Protoc 5: 550-560, 2010.

19. Sun S, Guo Z, Xiao X, et al: Isolation of mouse marrow mesenchymal progenitors by a novel and reliable method. Stem Cells 21: 527-535, 2003.

20. Guo Z, Li H, Li X, et al: In vitro characteristics and in vivo immunosuppressive activity of compact bone-derived murine mesenchymal progenitor cells. Stem Cells 24: 992-1000, 2006.

21. Maxson S, Lopez EA, Yoo D, Danilkovitch-Miagkova A and Leroux MA: Concise review: role of mesenchymal stem cells in wound repair. Stem Cells Transl Med 1: 142-149, 2012.

22. Wu Y, Chen L, Scott PG and Tredget EE: Mesenchymal stem cells enhance wound healing through differentiation and angiogenesis. Stem Cells 25: 2648-2659, 2007.

23. Cubas R, Li M, Chen C and Yao Q: Trop2: a possible therapeutic target for late stage epithelial carcinomas. Biochim Biophys Acta 1796: 309-314, 2009. 
24. Yu FX, Sun HQ, Janmey PA and Yin HL: Identification of a polyphosphoinositide-binding sequence in an actin monomerbinding domain of gelsolin. J Biol Chem 267: 14616-14621, 1992.

25. Linnenbach AJ, Seng BA, Wu S, et al: Retroposition in a family of carcinoma-associated antigen genes. Mol Cell Biol 13: $1507-1515,1993$.

26. El Sewedy T, Fornaro M and Alberti S: Cloning of the murine TROP2 gene: conservation of a PIP2-binding sequence in the cytoplasmic domain of TROP-2. Int J Cancer 75: 324-330, 1998.

27. Taniguchi CM, Tran TT, Kondo T, et al: Phosphoinositide 3-kinase regulatory subunit p85alpha suppresses insulin action via positive regulation of PTEN. Proc Natl Acad Sci USA 103: 12093-12097, 2006

28. Wan X, Dennis AT, Obejero-Paz C, et al: Oxidative inactivation of the lipid phosphatase and tensin homolog on chromosome ten (PTEN) as a novel mechanism of acquired long QT syndrome. J Biol Chem 286: 2843-2852, 2011.

29. Datta SR, Dudek H, Tao X, et al: Akt phosphorylation of BAD couples survival signals to the cell-intrinsic death machinery. Cell 91: 231-241, 1997.

30. Brunet A, Bonni A, Zigmond MJ, et al: Akt promotes cell survival by phosphorylating and inhibiting a Forkhead transcription factor. Cell 96: 857-868, 1999.
31. Song G, Ouyang G and Bao S: The activation of Akt/PKB signaling pathway and cell survival. J Cell Mol Med 9: 59-71, 2005.

32. Re RN and Cook JL: Senescence, apoptosis, and stem cell biology: the rationale for an expanded view of intracrine action. Am J Physiol Heart Circ Physiol 297: H893-H901, 2009.

33. Sethe S, Scutt A and Stolzing A: Aging of mesenchymal stem cells. Ageing Res Rev 5: 91-116, 2006.

34. Lindsley CW: The Akt/PKB family of protein kinases: a review of small molecule inhibitors and progress towards target validation: a 2009 update. Curr Top Med Chem 10: 458-477, 2010.

35. Litvinov SV, Velders MP, Bakker HA, Fleuren GJ and Warnaar SO: Ep-CAM: a human epithelial antigen is a homophilic cell-cell adhesion molecule. J Cell Biol 125: 437-446, 1994.

36. Maetzel D, Denzel S, Mack B, et al: Nuclear signalling by tumour-associated antigen EpCAM. Nat Cell Biol 11: 162-171, 2009.

37. Wang J, Zhang K, Grabowska D, et al: Loss of Trop2 promotes carcinogenesis and features of epithelial to mesenchymal transition in squamous cell carcinoma. Mol Cancer Res 9: 1686-1695, 2011. 